\title{
ON THE INVARIANTS OF A VECTOR SUBSPACE OF A VECTOR SPACE OVER A FIELD OF CHARACTERISTIC TWO
}

\author{
VERA PLESS
}

1. Introduction. Witt's theorem is concerned with the extension of an isometry between subspaces to an isometry on the whole space. The most general form of Witt's theorem is Theorem 1.2.1 in Wall [3]. Theorem 1 of this paper extends Theorem 1.2.1 and is identical to it in case the characteristic of the division ring is not 2. Theorem 2 is a variant of Theorem 1 . Theorems 1 and 2 are concerned with sesquilinear forms. Theorems 3 and 4 are concerned with bilinear forms on a finite dimensional vector space over a field of characteristic 2. Theorem 3 gives necessary and sufficient conditions for two (possibly degenerate) forms to be equivalent. Theorem 4 gives necessary and sufficient conditions for two subspaces to be equivalent.

The original results of this paper were based on results in Dieudonné [1]. However, the referee kindly pointed out that the proofs can be simplified and some of the results generalized by using results in Wall [3]. In particular he pointed out that Wall's proof is valid for the results stated in Theorem 1 as the restrictions contained in Theorem 1.2.1, are not necessary. He also suggested the variant on Theorem 1 which is Theorem 2. The proof of Theorem 4 has been considerably simplified by the use of Theorem 2 . I wish to thank the referee for these suggestions as it allows me to present these results in a more elegant and simplified form.

I also wish to thank Professor A. M. Gleason for stimulating discussions and advice, and Mr. E. Prange for discussions and for pointing out to me a proof of a weaker version of Theorem 1.

2. Notation. Let $V$ be a vector space of possibly infinite dimension over a division ring $D$ with a fixed involutory anti-automorphism $J$, that is, a one-to-one mapping $\alpha \rightarrow \alpha^{J}$ of $D$ onto itself such that $(\alpha+\beta)^{J}=\alpha^{J}+\beta^{J},(\alpha \beta)^{J}=\beta^{J} \alpha^{J}$, and $\alpha^{J^{2}}=\alpha$. An Hermitian (skewHermitian) sesquilinear form on $V$ is a mapping $f: V \times V \rightarrow D$ such that $f(x, y)$ is linear in $x$ for each fixed $y$ and $f(y, x)=f(x, y)^{J}(f(y, x)$ $\left.=-f(x, y)^{J}\right)$. If the characteristic of $D$ is two, the distinction between Hermitian and skew-Hermitian forms disappears.

Two forms $f_{1}$ and $f_{2}$ are called equivalent if there is a linear trans-

Received by the editors June 8, 1964 and, in revised form, September 28, 1964. 
formation $\sigma$ of $V_{1}$ onto $V_{2}$ with the property that

$$
f_{1}(x, y)=f_{2}(\sigma(x), \sigma(y))
$$

for all $x$ and $y$ in $V_{1}$; and $\sigma$ is called an isometry.

If $W \subset V, W^{\perp}$ is the set of all $y$ in $V$ such that $f(x, y)=0$ for all $x$ in $W$.

A form $f$ is called nondegenerate if $V^{\perp}=0$. Otherwise $f$ is called degenerate.

3. Witt's theorem. Let $f$ be a nondegenerate Hermitian or skewHermitian form on $V$. The set of all isometries of $V$ onto itself form the unitary group $U(f)$. If $\sigma$ is in $U(f)$ and the range of $I-\sigma$ is finite dimensional, then $\sigma$ is said to be finite dimensional. The finite dimensional elements of $U(f)$ form a normal subgroup of $U_{\phi}(f)$.

If $x$ is in $V$, we call $x$ trace-valued if $f(x, x)=\lambda+\epsilon \lambda^{J}, \epsilon= \pm 1$, for some $\lambda$ in $D$. By Lemma 1.2.1 in [3] the set of trace-valued vectors in $V$ forms a subspace $V^{r}$ of $V$. If the characteristic of $D$ is not $2, V^{r}=V$.

The most general form of Witt's theorem is Wall's Theorem 1.2.1 [3] which we now state.

Theorem 1.2.1 (WITt). Let $W_{1}, W_{2}$ be finite dimensional subspaces of $V^{\tau}$ such that $W_{1} \cap\left(V^{\tau}\right)^{\perp}=W_{2} \cap\left(V^{\tau}\right)^{\perp}=\{0\}$. Then every isometry $\sigma$ of $W_{1}$ onto $W_{2}$ can be extended to an element of $U_{\phi}(f)$.

The following is an extension of the preceding theorem and a generalization of the characteristic two case in [2].

THEOREM 1 (WITT). Let $W_{1}, W_{2}$ be finite dimensional subspaces of $V$ such that $W_{1} \cap\left(V^{\tau}\right)^{\perp}=W_{2} \cap\left(V^{\tau}\right)^{\perp}$. Then every isometry of $W_{1}$ onto $W_{2}$ which is the identity on $W_{1} \cap\left(V^{\tau}\right)^{\perp}$ can be extended to an element of $U_{\phi}(f)$.

Proof. By Lemma 1.2.2, Corollary [3], every element in $U(f)$ leaves $\left(V^{\tau}\right)^{\perp}$ pointwise invariant. Hence it is enough to be able to extend the isometry to an element of $U_{\phi}(f)$ under the assumption that $W_{1} \cap\left(V^{\tau}\right)^{\perp}=W_{2} \cap\left(V^{\tau}\right)^{\perp}=\{0\}$. This can be proved by following Wall's proof of Theorem 1.2.1 and deleting the restriction that $W_{1}, W_{2} \subset V^{\tau}$. This restriction is nowhere needed in the proof. Q.E.D.

If $W_{1}$ and $W_{2}$ are subspaces of $V$ and $W_{1}$ is isometric to $W_{2}$ by an isometry in $U_{\phi}(f)$ we will call $W_{1}$ and $W_{2}$ equivalent. Theorem 2 is a variant of Theorem 1.

Theorem 2. If $W_{1}$ and $W_{2}$ are finite dimensional subspaces of $V$, then $W_{1}$ is equivalent to $W_{2}$ if, and only if, (1) $W_{1} \cap\left(V^{\tau}\right)^{\perp}=W_{2} \cap\left(V^{\tau}\right)^{\perp}$ and (2) $W_{1}$ is isometric to $W_{2}$. 
Proof. The first condition is necessary by Lemma 1.2.2, Corollary in [3]. To prove the sufficiency we need only show that there is an isometry $\omega$ sending $W_{1}$ onto $W_{2}$ which sends $W_{1} \cap\left(V^{r}\right)^{\perp}$ on to $W_{2} \cap\left(V^{\tau}\right)^{\perp}$. Then $\omega$ would send a complement of $W_{1} \cap\left(V^{r}\right)^{\perp}$ in $W_{1}$ onto a complement of $W_{2} \cap\left(V^{\tau}\right)^{\perp}$ in $W_{2}$. Hence by the proof of Theorem 1 , there exists a $\sigma$ in $U_{\phi}(f)$ sending $W_{1}$ on to $W_{2}$.

To establish the existence of $\omega$, let $\rho$ be an isometry of $W_{1}$ onto $W_{2}$ such that the subspace $X=\left\{x \mid x\right.$ is in $W_{1} \cap\left(V^{r}\right)^{\perp}$ and $\rho(x)$ is in $\left.W_{2} \cap\left(V^{\tau}\right)^{\perp}\right\}$ have as large a dimension as possible. We assert $X$ equals $W_{1} \cap\left(V^{\tau}\right)^{\perp}$. We will prove this by contradiction. Suppose that $X$ does not equal $W_{1} \cap\left(V^{\tau}\right)^{\perp}$. Then there is an $a$ in $W_{1} \cap\left(V^{\tau}\right)^{\perp}$ with $\rho(a)$ not in $W_{2} \cap\left(V^{\tau}\right)^{\perp}$. Hence it is possible to find an $a$ such that in addition to $a \in W_{1} \cap\left(V^{\tau}\right)^{\perp}, \rho(a) \notin W_{2} \cap\left(V^{\tau}\right)^{\perp}$, we have $a=\rho(b)$ where $b \in W_{1}, b \in W_{1} \cap\left(V^{\tau}\right)^{\perp}$. Let $Y^{\prime}$ be the subspace generated by $a$ and $b$. Then $\operatorname{dim} Y^{\prime}=2$ and $X \cap Y^{\prime}=0$. Hence we can choose a complement $Y$ of $Y^{\prime}$ in $W_{1}$ such that $X \subset Y$.

Now we define a new isometry $\psi$ of $W_{1}$ onto $W_{2}$ as follows. $\psi(x)$ $=\rho(x)$ for $x$ in $Y \cdot \psi(a)=a \cdot \psi(b)=\rho(a)$. If $\psi$ is indeed an isometry we will have our contradiction. Clearly $\psi$ is one-to-one so we have to verify that $f(\psi(x), a)=f(x, a)$ and $f(\psi(x), \rho(a))=f(x, b)$ for all $x$ in $W_{1}$. By Lemma 1.2.2, Corollary [3], $\rho(x)-x$ is in $V^{\tau}$ for all $x$ in $V$ so that $f(\rho(x), a)=f(x, a)$ for all $x$ in $W_{1}$. If $x$ is in $Y, f(\psi(x), a)$ $=f(\rho(x), a)=f(x, a)$. It can be shown that $f(\psi(b), a)=f(b, a)$ also, so that $f(\psi(x), a)=f(x, a)$ for all $x$ in $W_{1}$. For $x$ in $Y, f(\psi(x), \rho(a))$ $=f(\rho(x), \rho(a))=f(x, a)=f(\rho(x), a)=f(\rho(x), \rho(b))=f(x, b)$. It can also be shown that $f(\psi(a), \rho(a))=f(a, b)$, so that $f(\psi(x), \rho(a))=f(x, b)$ for all $x$ in $W_{1}$. Q.E.D.

A subspace $W \subset V$ is called isotropic if $f(x, y)=0$ for all $x$ and $y$ in $W$.

CoROLlaRy 2.1. If $W_{1}$ and $W_{2}$ are two isotropic subspaces of $V$ such that $W_{1} \cap\left(V^{\tau}\right)^{\perp}=W_{2} \cap\left(V^{\tau}\right)^{\perp}, W_{1}$ and $W_{2}$ are equivalent.

4. Invariants. In this section we assume that $V$ is finite dimensional, $J$ is the identity, and $D$ is a field of characteristic two. Under these assumptions $f$ is a nondegenerate symmetric bilinear form, and $V^{\tau}$ is the set of all $x$ such that $f(x, x)=0$. Let $m=\operatorname{dim} V$.

Since $\lambda \rightarrow \lambda^{2}$ is an automorphism of $D$ into $D^{2}$, the mapping $\theta: x$ $\rightarrow f(x, x)$ is a semi-linear transformation of $V$ into the vector space $D$ over the field $D^{2}$. Let $W=\theta(V)$. Clearly $W$ is a subspace of $D$ over $D^{2}$. $V^{\tau}$ equals $\theta^{-1}(0)$. Let $U=\theta\left(\left(V^{\tau}\right)^{\perp}\right)$ and let $l=\operatorname{dim} U$.

Corollary 2.2. Any isotropic space is contained in an isotropic space of maximal dimension $\nu$. In addition $\nu=(m-l) / 2$. 
Proof. Given any isotropic space $U$, we can find a subspace $U^{\prime}$ of a maximal isotropic space $M$ such that $U \cap\left(V^{\tau}\right)^{\perp}=U^{\prime} \cap\left(V^{\tau}\right)^{\perp}$. Hence there is a $\sigma$ in $U(f)$ such that $\sigma(U)=U^{\prime}$ and $U$ is thus contained in the maximal isotropic space $\sigma^{-1}(M)$.

It can be shown that the direct sum of a maximal isotropic space in a complement of $V^{\tau} \cap\left(V^{\tau}\right)^{\perp}$ in $V^{\tau}$ and $V^{\tau} \cap\left(V^{\tau}\right)^{\perp}$ is a maximal isotropic subspace of $V$. Hence,

$$
\begin{aligned}
\nu & =\operatorname{dim} V^{\tau} \cap\left(V^{\tau}\right)^{\perp}+\frac{\operatorname{dim} V^{\tau}-\operatorname{dim} V^{\tau} \cap\left(V^{\tau}\right)^{\perp}}{2} \\
& =\frac{\operatorname{dim} V^{\tau}+\operatorname{dim} V^{\tau} \cap\left(V^{\tau}\right)^{\perp}}{2} \\
& =\frac{m-\left(\operatorname{dim}\left(V^{\tau}\right)^{\perp}-\operatorname{dim} V^{\tau} \cap\left(V^{\tau}\right)^{\perp}\right)}{2} \\
& =\frac{m-l}{2} .
\end{aligned}
$$

On p. 51 of [3], Wall has shown that $\langle f(x, x), f(y, y)\rangle=f(x, y)$ for $x$ and $y$ in $\left(V^{\tau}\right)^{\perp}$ uniquely defines a function $\gamma=\langle\lambda, \mu\rangle$ of the variables $\lambda, \mu$ in $U$. Lemma 3.4.2 of [3] states that two nondegenerate forms are equivalent if, and only if, they have the same $W, U$, and $\gamma$. If $f$ is a degenerate form let $d(f)=\operatorname{dim} V \cap V^{\perp}$. Then it follows that two (possibly degenerate) forms are equivalent if, and only if, they have the same $W, U, \gamma$, and $d$.

If $f_{1}$ and $f_{2}$ are two forms on $V$, let $V_{1}^{\tau}$ denote the $V^{\tau}$ for $f_{1}$ and $V_{2}^{\tau}$ denote the $V^{r}$ for $f_{2}$.

TheOREM 3. Two (possibly degenerate) forms $f_{1}$ and $f_{2}$ are equivalent if, and only if,

(1) $\left\{f_{1}(x, x)\right\}=\left\{f_{2}(x, x)\right\}$ and

(2) $\left(V_{1}^{\tau}\right)^{\perp}$ is isometric to $\left(V_{2}^{\tau}\right)^{\perp}$.

Proof. By the above discussion on $W, U, \gamma$, and $d$, it is enough to show that if two forms $f_{1}$ and $f_{2}$ satisfy conditions (1) and (2) they have the same $W, U, \gamma$, and $d$.

Clearly if $f_{1}$ and $f_{2}$ satisfy condition (1) they have the same $W$.

Also if $f_{1}$ and $f_{2}$ satisfy condition (2) it is not hard to see that they must have the same $U$ and $\gamma$.

To see that $f_{1}$ and $f_{2}$ have the same $d$ note that

$$
\begin{aligned}
d\left(f_{i}\right) & =\operatorname{dim} V_{i}^{\tau}+\operatorname{dim}\left(V_{i}^{\tau}\right)^{\perp}-\operatorname{dim} V \\
& =m-\operatorname{dim} W+\operatorname{dim}\left(V_{i}^{\tau}\right)^{\perp}-m \\
& =\operatorname{dim}\left(V_{i}^{\tau}\right)^{\perp}-\operatorname{dim} W . \quad \text { Q.E.D. }
\end{aligned} \quad i=1,2
$$


In [2] it was shown that any two nondegenerate forms $f_{1}$ and $f_{2}$ are equivalent under the condition that $f_{1}(x, x)$ and $f_{2}(x, x)$ both take their values in a perfect subfield of $D$. The next corollary shows that this is true for any two nondegenerate forms whose $W$ 's are the same one-dimensional subspace of $D$.

COROLlARY 3.1. If $f_{1}$ and $f_{2}$ are nondegenerate forms, each with a onedimensional $W$, then $f_{1}$ is equivalent to $f_{2}$ if, and only if, condition (1) holds.

Proof. Since $\operatorname{dim}\left(V_{1}^{\tau}\right)^{\perp}=\operatorname{dim} W=\operatorname{dim}\left(V_{2}^{\tau}\right)^{\perp}, \operatorname{dim}\left(V_{1}^{\tau}\right)^{\perp}=\operatorname{dim}\left(V_{2}^{\tau}\right)^{\perp}$ $=1$. Hence $\left(V_{1}^{\tau}\right)^{\perp}$ is isometric to $\left(V_{2}^{\tau}\right)^{\perp}$ if, and only if, either both are isotropic or both are not isotropic. It is known (p. 50 of [3]) that $\operatorname{dim} \theta\left(\left(V_{1}^{\tau}\right)^{\perp}\right) \equiv m(2)$ and $\operatorname{dim} \theta\left(\left(V_{2}^{\tau}\right)^{\perp}\right) \equiv m(2)$ so that $\left(V_{1}^{\tau}\right)^{\perp}$ and $\left(V_{2}^{\tau}\right)^{\perp}$ will be isotropic when $m$ is even, not isotropic when $m$ is odd.

REMARK. If $f_{1}$ and $f_{2}$ are (possibly degenerate) forms, each with a one-dimensional $W$, then $f_{1}$ is equivalent to $f_{2}$ if, and only if condition (1) holds and $d\left(f_{1}\right)=d\left(f_{2}\right)$.

Theorem 4. Let $W_{1}, W_{2}$ be subspaces of $V$. Then $W_{1}$ is equivalent to $W_{2}$ if, and only if:

(1) $\operatorname{dim} W_{1}=\operatorname{dim} W_{2}$, and

(2) $W_{1} \cap\left(V^{\tau}\right)^{\perp}=W_{2} \cap\left(V^{\tau}\right)^{\perp}$, and

(3) $W_{1}^{\perp} \cap\left(V^{\tau}\right)^{\perp}=W_{2}^{\perp} \cap\left(V^{\tau}\right)^{\perp}$, and

(4) $\left(W_{1} \cap V^{\tau}\right)^{\perp} \cap W_{1}$ is isometric to $\left(W_{2} \cap V^{\tau}\right)^{\perp} \cap W_{2}$.

Proof. Conditions (2) and (3) are necessary by Lemma 1.2.2, Corollary [3]. Conditions (1) and (4) are obviously necessary.

To prove the sufficiency we will first show that conditions (1), (3), and (4) imply that $W_{1}$ is isometric to $W_{2}$. Then the theorem follows from condition (2) and Theorem 2. Theorem 3 shows that $W_{1}$ and $W_{2}$ are isometric. This follows since condition (4) gives us condition (2) in Theorem 3 immediately. Condition (3) is equivalent to $W_{1}+V^{r}$ $=W_{2}+V^{\tau}$ which implies condition (1) in Theorem 3 .

The next corollary shows that these conditions are somewhat simpler for the situation where $\operatorname{dim} W=1$ and is a generalization of Corollary 3.2 in [2].

Corollary 4.1. If $\operatorname{dim} W=1$, then two subspaces $W_{1}$ and $W_{2}$ of $V$ are equivalent if, and only if:

(1) $\operatorname{dim} W_{1}=\operatorname{dim} W_{2}$, and

(2) $\operatorname{dim} W_{1} \cap\left(V^{\tau}\right)^{\perp}=\operatorname{dim} W_{2} \cap\left(V^{\tau}\right)^{\perp}$, and

(3) $\operatorname{dim} W_{1} \cap V^{\tau}=\operatorname{dim} W_{2} \cap V^{\tau}$, and

(4) $\operatorname{dim} W_{1} \cap W_{1}^{\perp}=\operatorname{dim} W_{2} \cap W_{2}^{\perp}$. 
Proof. These conditions are necessary by Theorem 4 . The sufficiency is proved in a fashion similar to the proof of Theorem 4.

By (1) and (3) $\operatorname{dim} \theta\left(W_{1}\right)=\operatorname{dim} \theta\left(W_{2}\right)$. Since $\operatorname{dim} W=1$, either $\operatorname{dim} \theta\left(W_{1}\right)$ and $\operatorname{dim} \theta\left(W_{2}\right)$ are both 1 or both 0 . Since condition (3) implies $W_{1}+V^{\tau}=W_{2}+V^{\tau}$, if both dimensions are $1, W_{1}$ and $W_{2}$ are isometric by the Remark to Corollary 3.1. In case both dimensions are 0 , conditions (1) and (4) are the known conditions for two symplectic spaces to be isometric.

Noting that condition (2) implies $W_{1} \cap\left(V^{\tau}\right)^{\perp}=W_{2} \cap\left(V^{\tau}\right)^{\perp}$, Theorem 2 tells us that $W_{1}$ and $W_{2}$ are equivalent.

Remark. If $\left(V^{\tau}\right)^{\perp}=0$, Theorems 3 and 4 are known [1] theorems for nondegenerate, alternating forms on spaces over fields of any characteristic.

\section{REFERENCES}

1. J. Dieudonne, Sur les groupes classiques, Hermann, Paris, 1958.

2. V. Pless, On Witt's Theorem for nonalternating symmetric bilinear forms over a field of characteristic 2, Proc. Amer. Math. Soc. 15 (1964), 979-983.

3. G. E. Wall, On the conjugacy classes in the unitary, symplectic and orthogonal groups, J. Austral. Math. Soc. 3 (1963), 1-62.

Air Force Cambridge Research Laboratories, Bedford, Massachusetts 